\title{
Os componentes motor e visual de uma tarefa-dupla devem ser associados ou isolados durante o treinamento?
}

\author{
Should motor and visual components of a dual-task be \\ associated or separated during training?
}

\author{
Mariana Callil Voos', Gisele Braga Pinheiro², Luciana Olcerenko Ciccaa, \\ Andrêa Lázaro ${ }^{3}$, Luiz Eduardo Ribeiro do Valle, Maria Elisa Pimentel Piemonte ${ }^{5}$
}

1 Fisioterapeuta; doutoranda em Neurociências e Comportamento no IP/USP (Instituto de Psicologia da Universidade de São Paulo, São Paulo, SP)

2 Fisioterapeuta; doutoranda em Fisiologia no ICB/USP (Instituto de Ciências Biomédicas da USP)

3 Fisioterapeutas; pós-graduadas em Fisioterapia em Neurologia

${ }^{4}$ Médico; Prof. Dr. do ICB/USP

5 Fisioterapeuta; Profa. Dra. do Fofito/FMUSP (Depto. de Fonoaudiologia, Fisioterapia e Terapia Ocupacional da Faculdade de Medicina da Universidade de São Paulo)

ENDEREÇO PARA

CORRESPONDÊNCIA

Mariana Callil Voos

Fofito/ FMUSP

Rua Cipotânea 51 Cidade

Universitária

05360-160 São Paulo SP

e-mail:marivoos@usp.br
Resumo: As atividades diárias requerem o desempenho simultâneo de tarefas (tarefa-dupla), não estando claro se seu treino deve ser realizado com tarefas isoladas ou associadas. Este estudo visou verificar se a aquisição de uma tarefadupla ocorre por meio do treinamento de tarefas isoladas ou associadas. Vinte voluntárias foram submetidas a avaliação inicial, treinamento e avaliação final. Dez treinaram as tarefas associadas (TA) e as demais, isoladas (TI). A tarefa motora consistia na alternância de passos do chão a uma plataforma. A tarefa visual consistia na nomeação de dois estímulos (ônibus ou caminhão), um no centro e outro na periferia da tela do computador. $\mathrm{O}$ número de passos por segundo na ausência e na presença da tarefa visual, e o número de erros na tarefa visual na ausência e na presença da tarefa motora, foram contados e comparados estatisticamente. O grupo TA apresentou melhora tanto no desempenho motor (inicial 1,10 alternâncias/s, final 1,25 alternâncias/s, $p=0,028$ ), quanto no desempenho visual (inicial 9,3 erros, final: 6,9 erros, $p=0,039$ ). O mesmo não ocorreu com o grupo TI: a melhora motora não atingiu nível significativo, provavelmente devido à maior variabilidade no número de alternâncias de passo por segundo durante o treinamento, e não houve melhora visual significativa $(p=0,844)$. Portanto, o tipo de treinamento interferiu no desempenho. O melhor desempenho ocorreu após o treinamento da tarefa-dupla. Descritores: Análise e desempenho de tarefas; Atenção; Atividade motora; Percepção visual

ABSTRACT: The association of tasks (dual-task) has functional importance in daily activities; people usually have to learn new tasks. It is unclear whether tasks should be trained isolated or associated. This experiment aimed at verifying whether the best dual-task performance occurs after training isolated or associated tasks. Twenty volunteers underwent initial assessment, training, and final assessment; ten trained associated tasks (AT) and the others trained tasks separately (IT). The motor task consisted of alternating steps between the ground and a platform. The visual task measured the ability to name two visual stimuli displayed on a computer screen (bus or truck). The number of step alternations per second in the absence and presence of the visual task, and the number of errors in the visual task in the absence and presence of the motor task, were counted and statistically analysed. The AT group showed both motor (initial 1.10 alternations/s, final 1.25 alternations/s; $p=0.028$ ) and visual (initial 9.3 errors, final 6.9 errors; $p=0.039$ ) improvement in performance. The same did not occur to the IT group: motor improvement did not reach significance level, probably due to the higher variability in the number of step alternations per second during training, and no visual improvement was shown $(p=0.844)$. It may thus be said that the kind of training interfered on performance. The best performance occurred after dualtask training.

KEY WORDS: Attention; Motor activity; Task performance and analysis; Visual perception 


\section{INTRODUÇÃO}

Muitas atividades de vida diária requerem gerenciamento simultâneo de componentes visuais e motores: atravessar a rua, levar o cão para passear, fazer compras, entrar e sair do metrô... Essas tarefas não são aprendidas apenas na infância; durante toda a vida deparamo-nos com novas demandas: dirigir, usar um joystick ou palm-top. As pessoas têm diferentes graus de dificuldade nesse aprendizado, de acordo com idade, escolaridade, experiência prévia, doenças. Muitos indivíduos procuram um fisioterapeuta por terem dificuldade em alguma tarefa visual-motora.

Atividades que envolvem tarefas associadas são denominadas tarefasduplas. Idosos ${ }^{1}$, crianças $^{2}$, pacientes com Parkinson ${ }^{3}$, acidente vascular encefálico ${ }^{4}$, traumatismo crânio-encefálico ${ }^{5}$, lesão medular ${ }^{6}$ apresentam dificuldade em tarefas-duplas. É importante o fisioterapeuta conhecer a aquisição do desempenho de tarefas-duplas em indivíduos saudáveis e, posteriormente, em pacientes, para treinamento adequado.

Geralmente, a realização da tarefadupla é mais difícil que a realização das tarefas isoladamente. Isso ocorre quando há interferência entre tarefas, ou seja, ambas competem pela mesma classe de recursos de processamento de informação no sistema nervoso central (SNC). NesSa situação, há interação negativa entre tarefas: quanto mais atenção for alocada para a realização de uma tarefa, pior será o desempenho na outra. A demanda atencional reflete o grau de competição entre representações corticais sobrepostas das duas tarefas (hipótese do campo cortical)7. A eficiência na realização de uma das tarefas pode determinar o grau de interferência com a tarefa concorrente ${ }^{8}$.

Outra hipótese que explica o fenômeno da interferência é a de que existe um centro de decisão e seleção de resposta, chamado gargalo (bottleneck), o qual tem capacidade limitada e res- tringe o desempenho de tarefas concorrentes $^{9}$. Não se sabe em que região do SNC está situado o gargalo, mas há indícios de envolvimento do córtex pré-motor dorsal, córtex frontal, giro frontal inferior esquerdo e área motora suplementar ${ }^{10}$.

Com o treinamento, a interferência negativa entre as tarefas diminui ${ }^{11}$ e o desempenho na tarefa-dupla melhora. Há relatos de maior capacidade atencional em tarefas visuais, conforme as atividades prévias do indivíduo. Foi observado melhor desempenho atencional em pessoas que usavam jogos eletrônicos em comparação às que tinham outras atividades de lazer ${ }^{12}$. Pessoas não treinadas evoluíram com ganho no desempenho após treino com jogos eletrônicos. Portanto, o número de repetições da tarefa visual no experimento pode interferir nos resultados.

Estudos apontam ganho de desempenho com treinamento de uma tarefaúnica e usam a tarefa-dupla como prova de automatização ${ }^{13,14}$. Automatização é a habilidade de desempenhar uma tarefa com recursos atencionais mínimos e ocorre quando a tarefa foi muito praticada ${ }^{15}$. Para testar automatização, acrescenta-se uma tarefa concorrente antes e depois do treinamento, para verificar se há diminuição da interferência negativa entre as tarefas após a prática ${ }^{15}$.

Considerando-se que essa vertente da literatura apresenta resultados favoráveis na condição tarefa-dupla após o treinamento na condição tarefaúnica, esta poderia ser uma evidência de que treinar na condição tarefa-única melhoraria a condição tarefa-dupla. Brown e Bennett ${ }^{14}$ defendem a idéia de que é melhor primeiro automatizar uma tarefa e depois acrescentar outra como secundária. Os autores trabalharam com duas tarefas: uma temporal (pressionar o mouse a cada cinco segundos) e uma não-temporal (leitura de um texto espelhado). O treinamento da não-temporal minimizou a interferência desta sobre a tarefa temporal (efeito de atenuação) $)^{14}$.
Quando uma tarefa-única é treinada e o teste de automatização é realizado na condição tarefa-dupla, o componente introduzido para caracterizar a tarefa-dupla adota o papel de distrator, sendo considerado menos relevante que a tarefa treinada, tida como primária. Esse tipo de treinamento pode ser pouco adaptativo, já que é comum precisarmos realizar tarefas-duplas com componentes de mesma relevância, como carregar compras e descer uma escada ou desviar de obstáculos e andar na calçada. Isso significa que o paradigma de introdução da tarefa secundária após o treinamento da tarefa primária é bom para mensurar a automatização, mas talvez não seja a melhor forma de proporcionar um treinamento visando aquisição de uma tarefa cujos componentes tenham a mesma relevância.

Embora muitos estudos tenham abordado o desempenho de tarefasduplas na literatura ${ }^{8-12,14}$, poucos realizaram a comparação do treinamento de tarefa-única $X$ tarefadupla ${ }^{16,17}$ e nenhum teve como objetivo determinar qual a melhor forma de treinar uma tarefa-dupla: se separandoa em duas tarefas e associando os componentes ao final do treinamento, ou mantendo a condição tarefa-dupla desde o início.

Melzer e Oddsson ${ }^{16}$ estudaram o treinamento da estratégia do passo na condição tarefa-única (dar o passo após um toque no calcanhar, realizado pelo examinador) e tarefa-dupla (realizar a tarefa inicial concomitante ao teste de Stroop) e verificaram que o desempenho (tempo de iniciação do passo) de voluntários jovens melhorou após o treinamento na condição tarefaúnica, mas não melhorou na condição tarefa-dupla. Os autores explicam esse resultado em função da menor variabilidade da condição tarefa-única, que poderia ter contribuído para a significância na comparação antes $X$ depois do treinamento: como a variabilidade da condição tarefa-dupla foi maior, o desempenho não atingiu níveis significativos. Porém, também é possível que o número de repetições tenha sido 
inadequado para obtenção de melhora na condição tarefa-dupla, ou que ambas as condições sejam muito fáceis para jovens e que realmente os achados estatísticos sejam apenas decorrentes de diferentes variabilidades. Talvez esse estudo não seja suficiente para concluir sobre a melhor forma de treinamento de uma tarefa-dupla.

A análise dos dados de Melzer e Oddsson ${ }^{16}$ tomando como base a teoria do gargalo e a hipótese do campo cortical permite concluir que a demanda atencional imposta pela tarefadupla foi maior que a capacidade de gerenciamento do SNC. Porém, essas teorias são insuficientes para explicar os resultados de Erickson et al. ${ }^{17}$, os quais compararam tarefa-única e tarefa-dupla trabalhando com ressonância magnética funcional e verificaram que uma tarefa-única é gerenciada por áreas diferentes de uma tarefa-dupla. Após o treinamento de uma tarefadupla, surge uma ativação pré-frontal dorso-lateral. Isso indica que, quando uma habilidade é treinada na condição tarefa-dupla, emerge uma nova capacidade de divisão de atenção, gerenciada pelo córtex pré-frontal dorsolateral. O mesmo não ocorre durante o treinamento isolado dos subcomponentes da tarefa.

Experimentos anteriores aos de Erickson et al. ${ }^{17}$ alegavam que um número menor de estruturas encefálicas seria necessário para gerenciar uma tarefa já treinada. Erickson et al. ${ }^{17}$ afirmam que, embora estruturas responsáveis pelo controle sensório-motor de fato estejam menos ativas após o treinamento, estruturas responsáveis por funções executivas (como atenção, tomada de decisão, seleção de resposta) poderiam estar mais ativas.

Este estudo visou determinar se, para a melhor aquisição de uma tarefadupla visual-motora, o treinamento mais efetivo seria por meio da repetição de blocos alternados das tarefas isoladas (visual $\rightarrow$ motora $\rightarrow$ visual $\rightarrow$ motora) ou da repetição de blocos das tarefas associadas (visual + motora $\rightarrow$ visual + motora)
Caso o melhor desempenho fosse constatado para o grupo que treinou as tarefas isoladas, nossos dados estariam de acordo com a hipótese de que, treinando-se os componentes de cada tarefa isoladamente, a demanda atencional para a execução de cada um diminuiria, ou seja, cada um seria automatizado e a condição tarefadupla melhoraria. Caso o melhor desempenho ocorresse para o grupo que treinou as tarefas associadas, a hipótese de que o treinamento de uma tarefa-dupla desenvolve uma nova habilidade de gerenciar tarefas simultâneas seria contemplada. Caso não houvesse diferença entre os grupos (ambos melhores após o treinamento ou nenhum efeito do treinamento), não haveria possibilidade de concluir qual hipótese seria a mais adequada.

\section{METODOLOGIA}

O estudo foi realizado em uma sala com isolamento acústico e iluminação reduzida, no Instituto de Ciências Biomédicas da Universidade de São Paulo (ICB/USP).

Participantes: 20 mulheres, alunas da Faculdade de Fisioterapia da USP, divididas em dois grupos de 10 . Os critérios de inclusão foram: ter de 20 a 30 anos, ser destra, apresentar de 15 a 23 anos de escolaridade, não ter experiência prévia com jogos eletrônicos ou experimentos semelhantes a este, ter visão normal ou corrigida por lentes, e consentir em participar mediante assinatura de um termo de consentimento aprovado pelo Comitê de Ética do ICB/USP.

Material: computador com monitor comum com tela de 14", com o aplicativo MEL2; plataforma de $10 \mathrm{~cm}$ de altura, fixa no chão, com cobertura antiderrapante; mesa de madeira com tampo de $110 \times 60 \mathrm{~cm}$ e $80 \mathrm{~cm}$ de altura; anteparos de madeira de alturas variadas para ajuste da altura do monitor conforme a altura da voluntária; ficha de avaliação.

\section{Tarefa visual}

A tarefa visual foi realizada com base na parte 2 do teste UFOV (Useful field of view test ${ }^{19}$. Consistia na nomeação de dois estímulos simultâneos, um no centro da tela do computador e outro na periferia (a dez graus do centro, em uma das oito possíveis posições demarcadas, numeradas de um a oito, em sentido horário), ambos com duração de 150 ms (Figura 1). A atividade visual era constituída por 32 tentativas, com duração total média de 2,5 minutos.

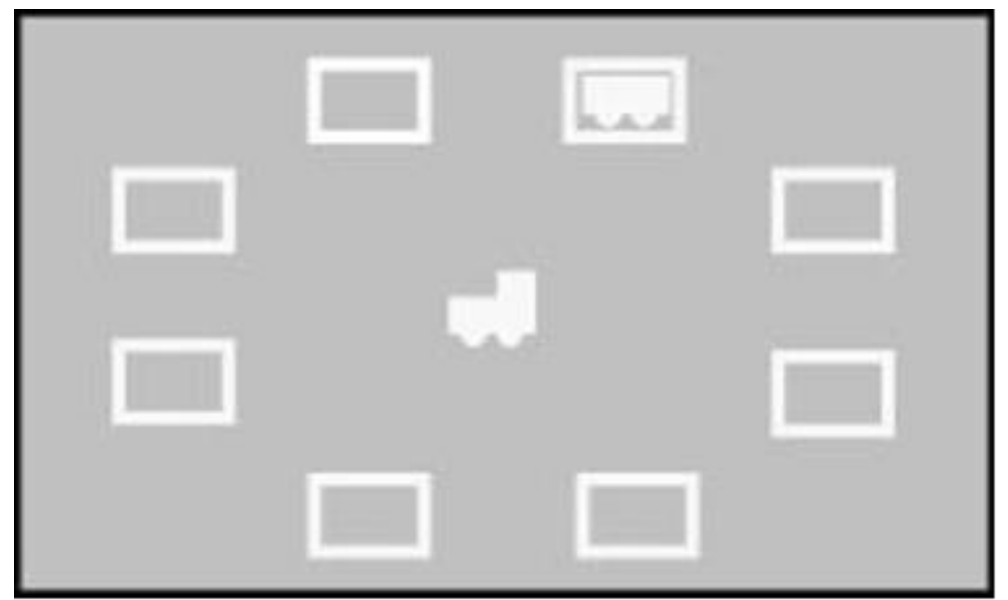

Figura 1 Tarefa visual: imagem apresentada na tela do computador; um ônibus ou um caminhão apareciam no centro e em um dos oito retângulos (estímulos simultâneos, de 150 ms) (Parte 2 do UFOV adaptada de Ball \& Owsley $\left.{ }^{19}\right)$. 
A tela, de fundo cor cinza, trazia no centro uma cruz de fixação e oito retângulos brancos circundando-a (luminância de $20 \mathrm{~cd} / \mathrm{m}^{2}, 2,2^{\circ} \mathrm{de}$ altura por $3,6^{\circ}$ de largura e $0,04^{\circ}$ de borda). Após algum tempo (1750 ms), aparecia um estímulo de duração de 150 ms no centro da tela (sobre a cruz) e outro, simultaneamente, dentro de um dos retângulos periféricos. Os estímulos podiam ser um caminhão ou um ônibus (ambos com luminância de $20 / \mathrm{m} 2,1,5^{\circ}$ de largura por $0,5^{\circ}$ de altura) (Figura 1).

A participante deveria responder oralmente se os estímulos eram iguais (dois ônibus ou dois caminhões) ou diferentes (um ônibus e um caminhão). Como se tratava de uma tarefa de acurácia, após a exibição dos alvos o programa era interrompido até que a voluntária respondesse. Após cada tentativa, um examinador, que não tinha acesso à tela do teste, registrava a resposta da voluntária com o mouse e uma nova tentativa era iniciada. O programa forneceu o número de erros de cada voluntária na tarefa visual.

Assim, dentro de cada bloco de 32 tentativas, havia as 32 situações possíveis, apresentadas aleatoriamente: ônibus no centro e ônibus em um dos oito retângulos (iguais); ônibus no centro e caminhão em um dos oito retângulos (diferentes); caminhão no centro e caminhão em um dos oito retângulos (iguais) e caminhão no centro e ônibus em um dos oito retângulos da periferia (diferentes).

A voluntária ficava em pé, a $70 \mathrm{~cm}$ da tela, cuja altura era ajustada para que os olhos ficassem no nível da cruz de fixação. A voluntária era orientada a manter o olhar na cruz durante todo o experimento, mas deveria deixar sua atenção difusa para conseguir reconhecer os estímulos apresentados no centro e na periferia da tela.

\section{Tarefa motora}

A tarefa motora consistia na alternância de passos entre o chão e uma plataforma localizada à frente da voluntária, durante o mesmo tempo de duração da tarefa visual. A voluntária deveria realizar a tarefa o mais rápido possível. Um segundo examinador registrou o número de alternâncias de passos e esse valor foi dividido pelo tempo, obtendo-se o número de alternâncias por segundo.

Antes de ser iniciada a sessão, as participantes receberam detalhada explicação oral sobre o experimento. Todas as voluntárias realizaram a avaliação, que consistia na realização das duas tarefas simultaneamente (tarefa-dupla). O treino diferiu entre os grupos: o grupo TI (dez voluntárias) realizou o treino com as tarefas isoladas, alternadas (visual $\rightarrow$ motora $\rightarrow$ visual $\rightarrow$ motora); o grupo TA (dez voluntárias) treinou as tarefas associadas (visual + motora $\rightarrow$ visual + motora). A reavaliação foi igual à avaliação inicial.

O número de passos por segundo na ausência e na presença da tarefa visual e o número de erros na tarefa visual na ausência e na presença da tarefa motora foram analisados estatisticamente (por ANOVA). A primeira análise foi: quatro condições da tarefa

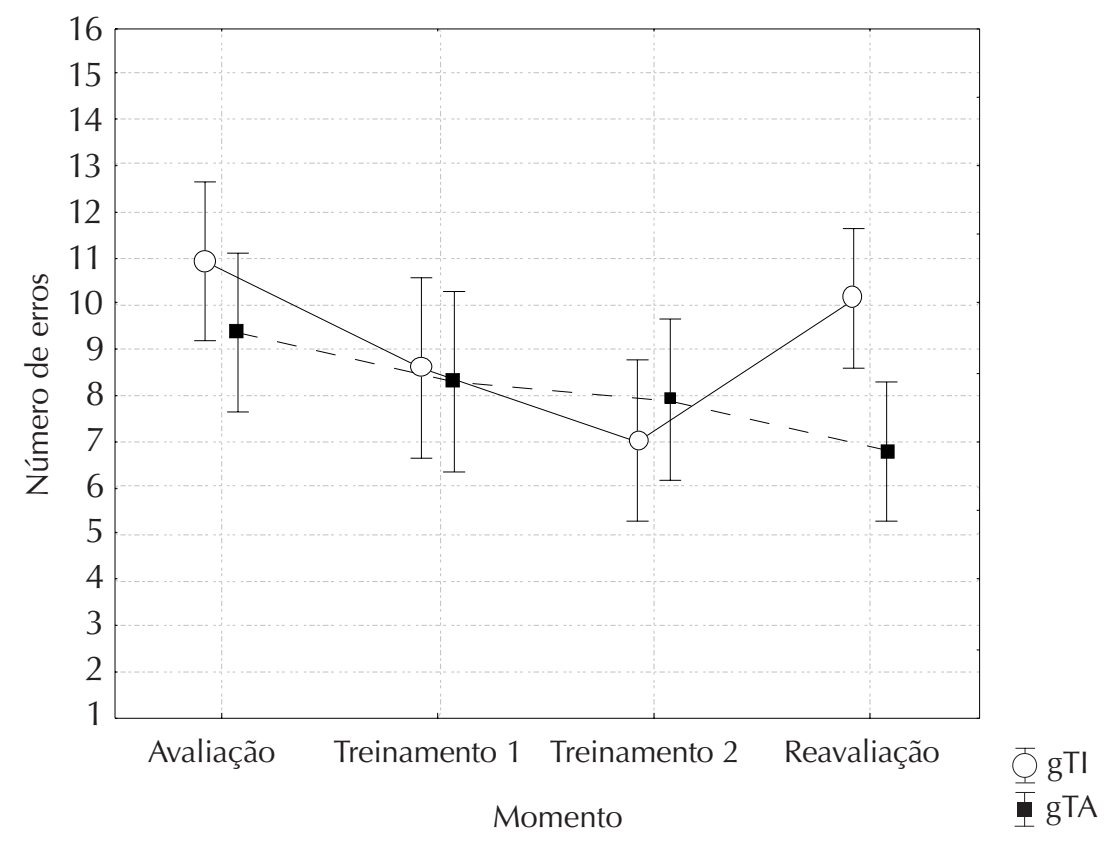

Figura 2 Efeito do treinamento sobre a tarefa visual: desempenho dos dois grupos na tarefa visual na avaliação, treinamento 1, treinamento 2 e reavaliação; $\mathrm{gTI}$ = grupo que treinou tarefas isoladas; gTA = grupo que treinou tarefas associadas visual - avaliação, treinamento 1 (tarefa visual e motora em um grupo ou tarefa visual + motora no outro), treinamento 2 (repetição do anterior) e reavaliação - X os dois grupos (TI e TA). A segunda análise foi: quatro condições da tarefa motora $X$ dois grupos (TI e TA).

\section{RESULTADOS}

\section{Tarefa visual}

Com relação ao número de erros, não houve diferença entre os grupos $\left[\mathrm{F}_{1,18}=1,952 ; p=1,209\right]$. Houve diferença entre condições $\left[F_{3,54}=7,932\right.$; $p<0,001]$, analisando-se os dois grupos em conjunto. A análise post hoc mostrou que a avaliação inicial foi significativamente pior que as demais medições (treinamento 1 , treinamento 2 e re-avaliação) ( $p=0,020 ; p<0,001 \mathrm{e}$ $p=0,020$, respectivamente), evidenciando o aprendizado ocorrido durante o experimento.

Houve interação momento $X$ grupo $\left[\mathrm{F}_{3,54}=5,068 ; p=0,004\right]$. A análise post 
hoc mostrou que, para o grupo que treinou as tarefas isoladas ( $\mathrm{TI})$, o número de erros no treinamento 2 foi menor quando comparado à avaliação inicial $(p<0,001)$, o que evidencia aprendizado durante a prática da tarefa visual isolada. Porém, o número de erros no treinamento 2 também foi menor que na reavaliação $(p=0,006)$. Logo, embora tenha havido melhora do desempenho na tarefa isolada, essa melhora não foi verificada na condição associada com relação ao desempenho inicial, que era o interesse principal neste estudo (inicial: 10,9 erros, final: 10,1 erros; $p=0,844$ ).

Para o grupo que treinou as tarefas associadas (gTA), a única comparação que atingiu nível significativo foi entre a avaliação e a reavaliação (inicial: 9,3 erros, final: 6,9 erros; $p=0,039$ ), logo, o número de erros na reavaliação foi significativamente menor que na avaliação inicial, sendo possível constatar que o treinamento da tarefa visual associada à motora possibilitou aprendizado da tarefa visual (Figura 2).

\section{Tarefa motora}

Com relação ao número de alternâncias de passo/segundo, não houve diferença entre os grupos $\left[\mathrm{F}_{1,18}=1,952\right.$; $p=0,179]$. Houve diferença entre os momentos $\left[\mathrm{F}_{3,54}=13,196 ; p<0,001\right]$, analisando-se os dois grupos em conjunto. A análise post hoc mostrou que a avaliação inicial foi significativamente pior que as demais (treinamento 1, treinamento 2 e reavaliação) ( $p<0,001$ para todas as comparações), evidenciando aprendizado com o treinamento.

Houve interação momento $X$ grupo $\left[\mathrm{F}_{3,54}=4,002 ; p=0,012\right] . \mathrm{A}$ análise post hoc mostrou que, para o grupo $\mathrm{TI}$, o número de alternâncias de passo no treinamento 1 e no treinamento 2 foi maior que na avaliação inicial $(p<0,001$ para as duas comparações), evidenciando aprendizado durante a prática da tarefa motora isolada. Porém, o número de alternâncias de passo na reavaliação não atingiu nível de significância com relação à avaliação

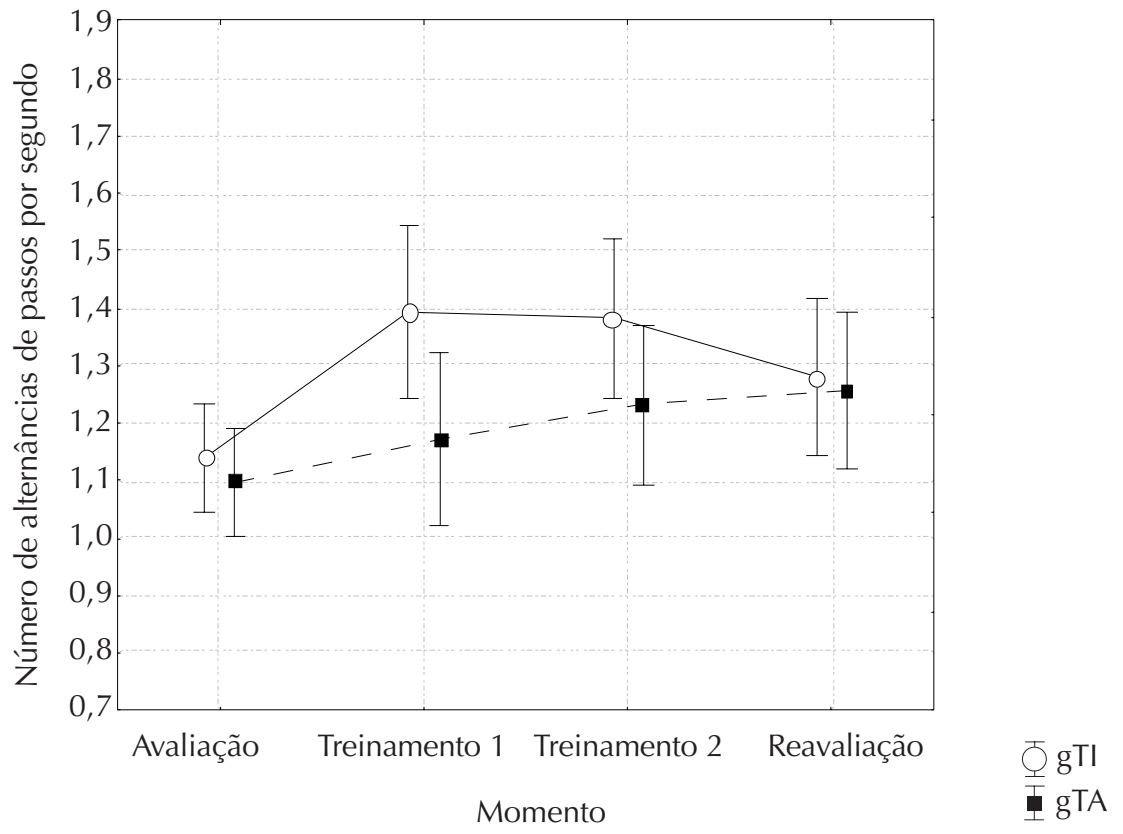

Figura 3 Efeito do treinamento sobre a tarefa motora: desempenho dos dois grupos na tarefa motora na avaliação, treinamento 1, treinamento 2 e reavaliação; $\mathrm{gTI}$ = grupo que treinou tarefas isoladas; gTA = grupo que treinou tarefas associadas

inicial $(p=0,075)$, de modo que a meIhora do grupo TI na condição tarefadupla não foi estatisticamente confirmada.

Para o grupo TA, a única comparação que atingiu nível significativo foi entre a avaliação e a reavaliação $(p=0,029)$, sendo o número de alternâncias de passo na reavaliação significativamente maior que na avaliação inicial. Esse dado confirmou o efeito positivo do treinamento da tarefa motora associada à visual (Figura 3).

Provavelmente, não foi possível verificar a melhora do grupo TI porque este apresentou valores superiores aos do TA nos treinamentos 1 e 2 e isso aumentou a variabilidade da amostra pois, numericamente, a diferença entre os grupos foi equivalente (gTI: inicial 1,12 alternâncias/s e final 1,27 alternâncias/s; gTA: inicial 1,10 alternâncias/s e final 1,25 alternâncias/s); ou seja, houve um aumento de 0,15 alternâncias por segundo em cada grupo.

\section{DISCUSSÃO}

Este estudo propõe novas reflexões quanto ao treinamento de uma tarefadupla. Enquanto a maioria dos trabaIhos até o momento utilizou a condição de tarefa-dupla apenas como prova de automatização, introduzindo a tarefa secundária como distrator ${ }^{8-11,13,14}$ este propõe o estudo do treinamento e da aquisição de uma tarefa-dupla. O treinamento na condição tarefa-dupla foi comparado ao treinamento na condição tarefa-única.

Os resultados divergem dos de Melzer e Oddsson ${ }^{16}$, que obtiveram melhor aproveitamento da condição tarefa-única para ganho de desempenho na estratégia do passo. Enquanto no estudo de Melzer e Oddsson o desempenho dos jovens não melhorou após o treinamento na condição tarefadupla, em nosso estudo a melhora na condição tarefa-dupla foi a que chegou a níveis significativos.

Como Melzer e Oddsson avaliaram 
jovens e idosos, talvez a tarefa tenha sido muito fácil para os jovens e o número de repetições tenha sido insuficiente para gerar aprendizado em universitários, que já partem geralmente de um bom desempenho. Os autores explicaram seus resultados em função da menor variabilidade da condição tarefa-única, que poderia ter contribuído para a significância na comparação antes $X$ depois do treinamento: como a variabilidade da condição tarefa-dupla foi maior, o desempenho não atingiu níveis significativos.

No presente estudo, ocorreu o oposto: o grupo que treinou as tarefas isoladas (gTI) apresentou aprendizado (aumento do número de alternâncias por segundo) nos treinamentos 1 e 2 , mas, embora tenha havido uma tendência, o número de alternâncias de passo na reavaliação não foi significativamente maior que na avaliação inicial. O fato de o grupo $\mathrm{TI}$ ter tido maior ganho nos treinamentos 1 e 2 fez com que aumentasse a variabilidade da amostra, não sendo possível a confirmação estatística da melhora apresentada que, numericamente, é igual entre os grupos. Apesar de esses dados parecerem inconclusivos, ao comparar os resultados obtidos no desempenho motor no presente experimento com os de Melzer e Oddsson, o fato de ter sido dado o mesmo peso à tarefa visual e à tarefa motora e de ter sido analisado também o desempenho visual (ao invés de considerarmos a tarefa visual apenas como um distrator) facilitou a interpretação dos presentes achados.

O teste visual utilizado exigiu atenção dividida para a comparação dos estímulos e seleção de resposta ("iguais" ou "diferentes"). Além da divisão de atenção entre os dois estímulos visuais, as voluntárias precisaram alocar recursos atencionais para a tarefa motora, realizando-a o mais rápido possível. Provavelmente, no presente estudo o teste visual foi mais difícil do que o utilizado por Melzer e Oddsson, pois as voluntárias partiram de um desempenho médio de 10 erros, num total de 32 tentativas. No estudo de Melzer e Oddsson houve efeito-teto no desempenho, portanto não foi possível constatar grande melhora. Neste estudo, com a tarefa visual utilizada, foi possível demonstrar que o treino na condição tarefa-dupla foi mais vantajoso para o aprendizado da tarefa visual (Figura 2).

Nossos resultados podem ser explicados com base nos achados de Erickson et al. ${ }^{17}$, que constataram que uma tarefa-única é gerenciada por áreas distintas de uma tarefa-dupla. Após o treinamento de uma tarefa-dupla, além das áreas ativadas na tarefa-única, Erickson et al. ${ }^{17}$ verificaram uma ativação pré-frontal dorso-lateral, decorrente da aquisição de uma nova capacidade de divisão de atenção. O mesmo não ocorreu durante o treinamento isolado dos subcomponentes da tarefa. Em nosso estudo, o treinamento na condição tarefa-dupla parece ter resultado numa nova habilidade de divisão de atenção entre as tarefas visual e motora, que não ocorreu no treinamento na condição tarefa-única.

Portanto, considerando-se que em nossas atividades de vida diária a condição tarefa-dupla é mais freqüente, parece ser mais interessante o treinamento de tarefas associadas. Não adianta treinarmos um indivíduo até que ele realize uma nova tarefa motora ou visual perfeitamente, acreditando que, com a diminuição da demanda atencional para cada tarefa, esse indivíduo será capaz de realizar a tarefa-dupla sem piora do desempenho.
A melhora da capacidade de dividir a atenção foi descrita por Green e Bavelier ${ }^{12}$ com o treinamento com jogos de ação. Todavia, o presente estudo apresenta uma contribuição que vai além dos achados de Green e Bavelier porque, enquanto estes avaliaram apenas a divisão de atenção espacial, no presente experimento, além da divisão da atenção em diferentes locais da tela do computador, conseguimos detectar e descrever a aquisição de uma habilidade de divisão de atenção visual e motora.

Uma limitação deste estudo é a participação apenas de mulheres jovens de alta escolaridade. Estudos futuros poderão verificar se esse comportamento é observado em indivíduos com outras características. Também destacamos o fato de as avaliações serem iguais a uma das formas de treinamento, que foi a que apresentou melhora. Em estudos futuros, serão aplicados testes de generalização, para verificar se a capacidade de divisão de atenção é transferida a outras habilidades. Os dados de Green e Bavelier ${ }^{12}$ e de Ball et al. ${ }^{19}$, que trabalharam com tarefas semelhantes à do presente experimento, constituem um forte indício de que essa generalização ocorra.

\section{CONCLUSÃO}

Para a melhor aquisição de uma tarefa-dupla visual-motora, concluímos que os componentes visual e motor devem ser treinados associados (visual+motora $\rightarrow$ visual+motora). É comum na prática fisioterapêutica a fragmentação de uma tarefa-dupla em subcomponentes simplificados, mas essa separação pode não resultar na habilidade futura de realização da tarefa-dupla. 


\section{REFERENCIAS}

1 Broman AT, West SK, Munoz B, Bandeen-Roche K, Rubin GS, Turano KA. Divided attention as a predictor of bumping while walking: the Salisbury eye evaluation. Invest Ophthalmol Vis Sci. 2004;45(9):2955-60.

2 Olivier I, Cuisinier R, Vaugoyeau M, Nougier V, Assaiante C. Dual-task study of cognitive and postural interference in 7-year-olds and adults. Neuroreport. 2007;18(8):817-21.

3 Yogev G, Giladi N, Peretz C, Springer S, Simon ES, Hausdorff JM. Dual tasking, gait rhythmicity and Parkinson's disease: which aspects of gait are attention demanding? Eur J Neurosci. 2005; 22:1248-56.

4 Brown LA, Sleik RJ, Winder TR. Attentional demands for postural control. Arch Phys Med Rehabil. 2002;83(12):1732-5.

5 Calvanio R, Williams R, Burke DT, Mello J, Lepak P, Al-Adawi $S$, et al. Acquired brain injury, visual attention and the useful visual field of view test: a pilot study. Arch Phys Med Rehabil. 2004;85:474-8.

6 Barbeau H, Fung J, Leroux A, Ladouceur M. A review of the adaptability and recovery of locomotion after spinal cord injury. Prog Brain Res. 2002;137:9-25.

7 Roland PE, Ziles K. Structural divisions and functional fields in the human cerebral cortex. Brain Res Brain Res Rev. 1998;26(2-3):87-105.

8 Matthews A, Garry MI, Martin F, Summers J. Neural correlates of performance trade-offs and dual-task interference in bimanual coordination: an ERP investigation. Neurosci Lett. 2006;400:172-6.

9 Pashler H. Dual-task interference in simple tasks: data and theory. Psychol Bull. 1994;116(2):220-44.
10 Marois R, Larson JM, Chun MM, Shima D. Responsespecific sources of dual-task interference in human pre-motor cortex. Psychol Res. 2006;70(6):436-47.

11 Wickens CD. Multiple resources performance prediction. Theor Issues Ergonom Sci. 2002;3:159-77.

12 Green CS, Bavelier D. Action video game modifies visual selective attention. Nature. 2003;423:534-7.

13 Beilock SL, Wierenga SA, Carr TH. Expertise, attention and memory in sensorimotor skill execution: impact of novel task constraints on dualtask performance and episodic memory. Q J Exp Psychol. 2002;55A(4):1211-40.

14 Brown SW, Bennett ED. The role of practice and automaticity in temporal and nontemporal dual-task performance. Psychol Res. 2002;66(1):80-9.

15 Ahissar M, Laiwand R, Hochstein S. Attentional demands following perceptual skill training. Psychol Sci. 2001;12(1):56-62.

16 Melzer I, Oddsson LIE. The effect of a cognitive task on voluntary step execution in healthy elderly and young individuals. J Am Geriatr Soc. 2004;52:1255-62.

17 Erickson KI, Colcombe SJ, Wadhwa R, Bherer L, Peterson MS, Scalf PE, et al. Training-induced functional activation changes in dual-task processing: an FMRI study. Cereb Cortex. 2007;17(1):192-204.

18 Puttemans V, Wenderoth N, Swinnen SP. Changes in brain activation during the acquisition of a multifrequency bimanual coordination task: from the cognitive stage to advanced levels of automaticity. J Neurosci. 2005;25(17):4270-8.

19 Ball K, Owsley C J. The useful field of view test: a new technique for evaluating age-related declines in visual function. Am Optom Assoc. 1993;64(1):71-9. 